\title{
On Achieving Optimal Degrees of Freedom of MIMO Cellular Networks Using Decomposition
}

\author{
Gokul Sridharan and Wei Yu \\ The Edward S. Rogers Sr. Department of Electrical and Computer Engineering \\ University of Toronto, Toronto, ON, M5S3G4, Canada \\ Email:gsridharan@comm.utoronto.ca, weiyu@comm.utoronto.ca
}

\begin{abstract}
This paper investigates the significance of decomposition-based schemes in achieving the optimal degrees of freedom (DoF) of multiple-input multiple-output (MIMO) cellular networks. We consider MIMO cellular networks with $G$ cells, $K$ users/cell with $M$ antennas at each user, and $N$ antennas at each base-station (BS). We assume all channels to be generic and time varying. We show that $\frac{K M N}{K M+N}$ DoF/cell can be achieved through one-sided decomposition of a MIMO cellular network (on the user side), followed by the use of an asymptotic interference alignment scheme. We then prove that such an approach achieves the optimal DoF whenever $\frac{M}{N}=\frac{1}{q}$, where $q \in\left\{\frac{1}{G-1}, \frac{1}{G-2}, \cdots, 1,2, \cdots,(G-1) K\right\}$. The optimality is proved using a new set of outer bounds on the DoF of a MIMO cellular network. Finally, we comment on the DoF achieved using two-sided decomposition, and the optimal DoF of cellular networks with single-antenna users and cellular networks with two cells.
\end{abstract}

\section{INTRODUCTION}

The study of degrees of freedom (DoF) has provided us with a better understanding of the role of interference in wireless networks. Starting with the single-input single-output (SISO) $K$-user interference channel [1], DoF of wireless networks such as the cellular networks [2], [3], and the wireless $X$ networks [4], [5] have been studied in great detail for the case when all nodes have the same number of antennas. Subsequently, the multiple-input multiple-output (MIMO) $K$ user interference channel having different number of antennas at the transmitters and receivers was studied in [6]-[8].

The techniques used to achieve the optimal DoF in these networks include the asymptotic interference alignment (AIA) scheme [1], which requires time/frequency varying channels and an infinite symbol extension, the rational dimensions framework [9], which works for constant channels and does not require any symbol extensions, and linear transmit/receive beamformer design over finite time/frequency extensions [1], [8]. While the asymptotic alignment schemes of [1] and [9] can be readily used to establish achievable DoF for a very broad set of wireless networks, designing linear transmit/receive beamformers needs significant customization to each wireless network.

This paper focuses on the DoF of MIMO cellular networks. While several outer bounds [10]-[12] and achievable schemes [12]-[17] are available, an exact characterization of the DoF for cellular networks with arbitrary number of cells and arbitrary number of users per cell is yet to emerge and is expected to be quite challenging. In this work, we provide a partial characterization of the DoF of MIMO cellular networks with $G$ cells, $K$ users per cell, $M$ antennas at each user and $N$ antennas at each base-station (BS). In particular, we study the achievable DoF using the AIA scheme proposed in [1] with the goal of establishing an inner bound on the DoF of a broad set of MIMO cellular networks.

The use of the AIA scheme [1] for MIMO networks requires the decomposition of multi-antenna nodes into multiple independent single-antenna nodes. Two-sided decomposition involves decomposing both transmitters and receivers into single-antenna nodes, while one-sided decomposition involves decomposing either the transmitters or the receivers. Once a network has been decomposed, the AIA scheme can be applied to get an inner bound on the DoF of the original network. Two-sided decomposition was first used to prove the achievability of optimal DoF of a $K$-user interference channel with the same number of antennas at each node [1]. This in turn showed that the network was two-side decomposable, i.e., no DoF are lost by decomposing multi-antenna nodes into single antenna nodes. Two-sided decomposition is also known to achieve the optimal DoF of MIMO cellular networks with same number of antennas at each node [3]. However, for wireless $X$ networks with same number of antennas at each node, two-sided decomposition is shown to be suboptimal and that one-sided decomposition achieves the optimal DoF [5].

While decomposition-based approaches are known to be optimal when all nodes have the same number of antennas, they are not always optimal when the number of antennas at the transmitters and receivers are different [6], [7], [18]. Recently, the optimality/suboptimality of decomposition based approaches is studied in greater detail for the $K$-user MIMO interference channel when $K \geq 4$ [18]. The optimality of the DoF of MIMO cellular networks that can be achieved using two-sided/one-sided decomposition are not known to the best of our knowledge.

In this paper we study the achievable DoF using one-sided and two-sided decomposition of MIMO cellular networks. In particular, we show that $\frac{K M N}{K M+N} \mathrm{DoF} / \mathrm{cell}$ can be achieved using one-sided decomposition when $(G-1) K M \geq N$. We also derive a new set of outer bounds on the DoF of MIMO cellular networks using a key insight from [4]. Through the inner and outer bounds we derive, we show that the optimal DoF of MIMO cellular networks with $\frac{M}{N}=\frac{1}{q}$ where $q \in$ 
$\left\{\frac{1}{G-1}, \frac{1}{G-2}, \cdots, 1,2, \cdots,(G-1) K\right\}$, is given by $\frac{M N}{K M+N}$ DoF/user.

\section{System ModeL}

We consider $G$ interfering cells with $K$ users in each cell. Each user is assumed to have $M$ antennas and each BS is assumed to have $N$ antennas. We use the index pair $(i, j)$ to denote the $j^{t h}$ user in the $i^{t h}$ cell. We denote the channel from the $l^{t h}$ user in the $j^{\text {th }}$ cell to the $i^{t h}$ BS as the $N \times M$ matrix $\mathbf{H}_{(j l, i)}$. We assume all channels to be generic and time varying. In the uplink, assuming the $l^{t h}$ user in the $j^{t h}$ cell transmits the $M \times 1$ signal vector $\mathbf{x}_{j l}$, the received signal at the $i^{t h} \mathrm{BS}$ is given by

$$
\mathbf{y}_{i}=\sum_{j=1}^{G} \sum_{l=1}^{K} \mathbf{H}_{(j l, i)} \mathbf{x}_{j l}+\mathbf{n}_{i}
$$

where $\mathbf{y}_{i}$ is an $N \times 1$ vector and $\mathbf{n}_{i}$ is the $N \times 1$ vector representing circular symmetric additive white Gaussian noise $\sim \mathcal{N}(\mathbf{0}, \mathbf{I})$. The received signal is defined similarly for the downlink.

\section{MAin Results}

\section{A. Achievable DoF using decomposition based schemes}

We are interested in the DoF/user that can be achieved in a MIMO cellular network using the AIA scheme proposed in [1]. This scheme was proposed for a network with singleantenna nodes and crucially relies on the commutativity of the time/frequency extended channels. Applying this scheme to a MIMO network requires us to decompose either the transmitters or the receivers, or both, into independent singleantenna nodes. When using the AIA scheme on the decomposed network, the DoF achieved per user in the original network is simply the sum of the DoFs achieved over the individual single-antenna nodes. We first consider two-sided decomposition as this results in a SISO network over which the AIA scheme can be used without any modifications. The following theorem states the achievable DoF using two-sided decomposition.

Theorem 3.1 For the $(G, K, M, N)$ cellular network, using two-sided decomposition, $\max \left(\frac{K M}{\left\lceil\frac{K M}{N}\right\rceil+1}, \frac{\left\lfloor\frac{K M}{N}\right\rfloor N}{\left\lfloor\frac{K M}{N}\right\rfloor+1}\right)$ DoF/cell are achievable.

Proof: The proof of this theorem follows from a direct application of the AIA scheme in [1]. We first decompose each of the $G N$-antenna BSs into $G N$ single-antenna BSs. A similar decomposition of the users gives $K M$ single-antenna users per cell. We divide the $K M$ single-antenna users in each cell into $N$ groups, each having $\approx(K M / N)$ users. If $\frac{K M}{N}$ is not an integer, we create fictitious single antenna users to get to $\left\lceil\frac{K M}{N}\right\rceil=W$ users per group. We then assign each group to one of the $N$ single-antenna BSs created within each cell. Each set of $W$ users assigned to a single-antenna BS are exclusively served by that BS. We have thus created a $G N$-cell SISO cellular network with $W$ users/cell. For such a network, it is known that $\frac{1}{W+1}$ DoF/user is optimal [3]. Thus, for the original network, using a two-sided decomposition, $\frac{K M}{W+1}$ DoF/cell are achieved.

Alternately, instead of adding fictitious users, we can consider selecting only $\left\lfloor\frac{K M}{N}\right\rfloor N$ single-antenna users so that each of the $N$ groups has $\left\lfloor\frac{K M}{N}\right\rfloor$ single-antenna users. Assigning each set of users to a single-antenna BS, we see that $\frac{\left\lfloor\frac{K M}{N}\right\rfloor N}{\left\lfloor\frac{K M}{N}\right\rfloor+1}$ DoF/cell can be achieved in the original MIMO cellular network.

The achievability of $\frac{\left\lfloor\frac{K M}{N}\right\rfloor N}{\left\lfloor\frac{K M}{N}\right\rfloor+1}$ DoF/cell for MIMO cellular networks is also discussed in [17]. However, it may not always be better to select only $\left\lfloor\frac{K M}{N}\right\rfloor$ single-antenna users per singleantenna BS. For example, when $N=15, M=1$ and $K=29\left(\left\lceil\frac{K M}{N}\right\rceil=2\right)$, it is clear that creating $N$ groups of 2 single-antenna users achieves 29/3 DoF/cell and is better than creating $N$ groups with just 1 user per group, in which case only 15/2 DoF/cell are achieved. On the other hand, when $K=16$, it is better to create groups with 1 user each $(15 / 2$ DoF/cell are achievable) as opposed to groups with 2 users (16/3 DoF/cell are achievable).

We note that when $N$ is a multiple of $K M, \frac{K M N}{K M+N}$ DoF/cell are achievable. We investigate the optimality of twosided decomposition in Section IV.

We now consider one-sided decomposition of MIMO cellular networks and specifically focus on decomposition at the user side which reduces the network to a cellular network with single-antenna users. The following lemma establishes the achievable DoF for a cellular network with single-antenna users and multi-antenna BSs.

Lemma 3.1 For the $(G, K, M, N)$ cellular network with $M=1, \frac{K N}{K+N}$ DoF/cell are achievable when $(G-1) K \geq N$.

Since one-sided decomposition of MIMO interference channels also results in a cellular network with single-antenna users, this is a direct consequence of the results in [6], [7]. The proof of this lemma is based on an extension of the AIA scheme to MIMO $K$-user interference channels [7]. We outline the key steps in Section V, where we consider achievability in the uplink. By duality of linear interference alignment, this also establishes achievability in the downlink.

We now state the achievable DoF for general MIMO cellular networks by decomposing each user into independent single-antenna users.

Theorem 3.2 For the $(G, K, M, N)$ cellular network, using one-sided decomposition, $\frac{K M N}{K M+N}$ DoF/cell are achievable when $(G-1) K M \geq N$.

Note that while we considered decomposing multi-antenna users into single-antenna users, we can alternately also consider decomposing the multi-antenna BSs. It can however be shown that the achievable DoF remains unchanged. Designing the achievable scheme is similar to [5] and is significantly more challenging. We do not present a proof here for brevity. 


\section{B. Outer bounds on DoF}

We now derive a new set of outer bounds on the DoF of MIMO cellular networks. The bounds are based on a result in [4], where MIMO wireless $X$ networks with $A$ transmitters and $B$ receivers are considered. By focusing on the set of messages originating from or intended for a transmitterreceiver pair and splitting the total messages in the network into $A B$ sets, the authors in [4] derive a bound on the DoF of this set of messages. Letting $d_{i, j}$ represent the DoF between the $i^{t h}$ transmitter and the $j^{t h}$ receiver, the following lemma presents the outer bound obtained in this manner.

Lemma 3.2 ([4]) In a wireless $X$ network with A transmitters and $B$ receivers, the DoF of all messages originating at the $a^{\text {th }}$ transmitter and the DoF of all the messages intended for the $b^{\text {th }}$ receiver are bounded by

$$
\sum_{i=1}^{B} d_{a, i}+\sum_{j=1}^{A} d_{j, b}-d_{a, b} \leq \max (M, N)
$$

where $M$ is the number of antennas at the $a^{\text {th }}$ transmitter and $N$ is the number of antennas at the $b^{\text {th }}$ receiver. By symmetry, this bound also holds when the direction of communication is reversed.

Before we proceed to establish outer bounds on the DoF of a MIMO cellular network, we define the set $\mathcal{Q}_{1}$ to be the set of $\{2,3, \cdots,(G-1) K\}$ and the set $\mathcal{Q}_{2}$ to be the set $\left\{\frac{1}{G-1}, \frac{1}{G-2}, \cdots, 1\right\}$. The following theorem presents an outer bound on the DoF.

Theorem 3.3 If a $(G, K, M, N)$ cellular network satisfies $M / N \leq 1 / q$, where $q \in \mathcal{Q}_{1} \cup \mathcal{Q}_{2}$, then $N /(K+q)$ is an outer bound on the DoF/user of that network.

Proof: To prove this theorem, we first note that a cellular network can be regarded as a wireless $X$ network with some messages set to zero. Further, Lemma 3.2 is applicable even when some messages are set to zero. We split the proof into two parts, where we first consider $q \in \mathcal{Q}_{1}$, followed by $q \in$ $\mathcal{Q}_{2}$.

When $q \in \mathcal{Q}_{1}$, consider a set of $q$ users that do not belong to the $i^{\text {th }}$ cell, denote this set as $\mathcal{U}_{\tilde{i q}}$, and allow this set of users to cooperate fully. Applying Lemma 3.2 to the $i^{t h} \mathrm{BS}$ and the set of users $\mathcal{U}_{\tilde{i} q}$, we get

$$
\sum_{j=1}^{K} d_{i j, i}+\sum_{(g, h) \in \mathcal{U}_{\tilde{i} q}} d_{g h, g} \leq \max (N, q M)=N
$$

By establishing similar bounds for all the other $\left(\begin{array}{c}(G-1) K \\ q\end{array}\right)$ sets of users and summing over all such bounds for a fixed $i$, we obtain

$$
\frac{(G-1) K}{q} \sum_{j=1}^{K} d_{i j, i}+\sum_{g=1, g \neq i}^{G} \sum_{h=1}^{K} d_{g h, g} \leq \frac{(G-1) K N}{q} .
$$

By summing over all such bounds for $i \in\{1,2, \cdots G\}$, we get

$$
\left(\frac{K}{q}+1\right) \sum_{i=1}^{G} \sum_{j=1}^{K} d_{i j, i} \leq \frac{G K N}{q} .
$$

Thus, the total DoF in the network is bounded by $\frac{G K N}{K+q}$. Hence, DoF/user $\leq \frac{N}{K+q}$ whenever $q \in \mathcal{Q}_{1}$.

When $q \in \mathcal{Q}_{2}$, we let $p=\frac{1}{q}$, and consider forming groups of $p$ BSs and letting them cooperate. Consider a set $\mathcal{B}_{\tilde{i} p}$ of $p$ BSs that does not include the $i^{\text {th }}$ BS. Applying Lemma 3.2 to the $j^{\text {th }}$ user in the $i^{t h}$ cell and the set of BSs $\mathcal{B}_{\tilde{i}}$, we get

$$
d_{i j, i}+\sum_{g \in \mathcal{B}_{\tilde{i} p}} \sum_{h=1}^{K} d_{g h, g} \leq \max (N p, M)=N p
$$

Summing over all $\left(\begin{array}{c}G-1 \\ p\end{array}\right)$ sets of $p$ BSs for a fixed $(i, j)$, followed by summing over all $(i, j)$ gives us

$$
\left(K+\frac{1}{p}\right) \sum_{i=1}^{G} \sum_{j=1}^{K} d_{i j, i} \leq \frac{G K N p}{p}
$$

Thus we see that even in this case DoF/user $\leq \frac{N p}{K p+1}=$ $\frac{N}{K+q}$. Note that whenever $\frac{M}{N}=\frac{1}{q}, \frac{N}{K+q}=\frac{M N}{K M+N}$.

Using a very similar proof technique, we can establish the following theorem for $\frac{M}{N}>\frac{1}{q}$.

Theorem 3.4 If a $(G, K, M, N)$ cellular network satisfies $M / N>1 / q$, where $q \in \mathcal{Q}_{1} \cup \mathcal{Q}_{2}$, then $M q /(K+q)$ is an outer bound on the DoF/user for that network.

In [10], outer bounds on the DoF for MIMO cellular network are derived which are also based on the idea of creating multiple message sets [4]. The DoF/user of a $(G, K, M, N)$ cellular network is shown to be bounded by

$$
\text { DoF/user } \leq \min \left(M, \frac{N}{K}, \frac{\max [K M,(G-1) N]}{K+G-1}, \frac{\max [N,(G-1) M]}{K+G-1}\right) \text {. }
$$

While it is difficult to compare this set of bounds and the bounds in Theorem 3.3 over all parameter values, we can show that under certain settings the bounds obtained in Theorem 3.3 are tighter. For example, since $q \in \mathcal{Q}_{1} \cup \mathcal{Q}_{2}$, let us fix $q=K$, and then set $\frac{M}{N}=\frac{1}{q}=\frac{1}{K}$. Further, let us assume that $(G-1)<K$. Under such conditions, (8) bounds the DoF/user by $\frac{M K}{K+G-1}$ while Theorem 3.3 states that DoF/user $\leq \frac{M}{2}$. Since we have assumed $K>G-1$ it is easy to see that the latter bound is tighter. In fact, in Section IV we show that outer bounds in Theorem 3.3 are indeed the optimal DoF/user whenever $\frac{M}{N}=\frac{1}{q}$ for some $q \in \mathcal{Q}_{1} \cup \mathcal{Q}_{2}$.

\section{Optimal DoF}

Using the results in Sections III-A and III-B, we establish conditions for the optimality of one-sided and two-sided decomposition of MIMO cellular networks in the following theorem. 
Theorem 4.1 The optimal DoF for any $(G, K, M, N)$ cellular network with $q \in \mathcal{Q}_{1} \cup \mathcal{Q}_{2}$ is $\frac{M N}{K M+N}$ DoF/user. This optimal DoF is achieved by one-sided decomposition on the user side. Two-sided decomposition achieves the optimal DoF if $q \in \mathcal{Q}_{1} \cup \mathcal{Q}_{2}$ and $\frac{K}{q} \in \mathbb{Z}^{+}$, where $\mathbb{Z}^{+}$denotes the set of positive integers.

This result follows immediately from Theorem 3.1, Theorem 3.2 and Theorem 3.3. We observe that this result is analogous to the results in [7] where it is shown that the $G$-user interference channel has $\frac{M N}{M+N}$ DoF/user whenever $\eta=\frac{\max (M, N)}{\min (M, N)}$ is an integer and $G>\eta$. It is easy to see that the results of [7] can be easily recovered from the above theorem by setting $K=1$ and letting $G$ represent the number of users in the interference channel.

Although two-sided decomposition is suboptimal compared to one-sided decomposition (except when $K / q \in \mathbb{Z}^{+}$and $q \in \mathcal{Q}_{1} \cup \mathcal{Q}_{2}$ ), unlike wireless $X$ networks, the reason here is due to the difficulty in partitioning the single-antenna users between the single-antenna BSs obtained after decomposition, and not because of inseparability of the received signal and interference as was the case with wireless $X$ networks [5]. We further note that unlike wireless $X$ networks, spatial scale invariance holds for two-sided decomposition of MIMO cellular networks. Finally, the asymptotic optimality of twosided decomposition is stated in the following corollary.

Corollary 4.1 For $(G, K, M, N)$ cellular networks, twosided decomposition is asymptotically optimal in the number of users and achieves $N$ DoF/cell as $K \rightarrow \infty$.

The result in Theorem 4.1 has important consequences for cellular networks with single antenna users. The following corollary describes the optimal DoF/user of any cellular network with single antenna users that satisfies $(G-1) K \geq N$.

Corollary 4.2 The optimal DoF of a $(G, K, M=1, N)$ network with $(G-1) K \geq N$, is $\frac{N}{K+N}$ DoF/user.

For example, this corollary states that a 3-cell network having 4 single-antenna users per cell and 4 antennas at each BS has $1 / 2 \mathrm{DoF} /$ user. This corollary also closes the gap between the upper and lower bounds on the DoF of 2-cell cellular networks with single-antenna users in [17] whenever $N<K$. In fact, combining the results in [17] for $N \geq K$ and Corollary 4.2 for $N<K$, the next theorem completely characterizes the DoF of 2-cell cellular networks with singleantenna users.

Theorem 4.2 The DoF of a two-cell cellular network with $K$ single-antenna users per cell and $N$ antennas at each $B S$ is given by

$$
\text { DoF/user }= \begin{cases}\frac{N}{N+K} & N<K \\ \frac{N}{2 K} & \quad K \leq N<2 K . \\ 1 & N \geq 2 K\end{cases}
$$

The optimal DoF are achieved through zero-forcing beamforming when $N \geq K$ and through asymptotic interference alignment when $N<K$.

\section{Proof of Lemma 3.1}

In this section, we present a brief proof of Lemma 3.1 based on [5], [7].

Consider a $r$ symbol extension of the channel so that each transmitter (user) has $r$ dimensions and each receiver (BS) has $r N$ dimensions. In the uplink, the received signal at the $i^{t h}$ BS for this extended channel can be written as

$$
\begin{aligned}
\hat{\mathbf{y}}_{i} & =\sum_{j=1, j \neq i}^{G} \sum_{l=1}^{K} \hat{\mathbf{H}}_{(j l, i)} \hat{\mathbf{x}}_{j l}+\hat{\mathbf{n}}_{j l} . \\
& =\sum_{j=1, j \neq i}^{G} \sum_{l=1}^{K}\left[\hat{\mathbf{H}}_{(j l, i 1)} \hat{\mathbf{H}}_{(j l, i 2)} \ldots \hat{\mathbf{H}}_{(j l, i N)}\right]^{T} \hat{\mathbf{x}}_{j l}+\hat{\mathbf{n}}_{j l}
\end{aligned}
$$

where $\hat{\mathbf{x}}_{j l}$ is the $r \times 1$ transmitted signal vector from the $l^{t h}$ user in the $j^{t h}$ cell, $\hat{\mathbf{H}}_{(j l, i c)}$ is the $r \times r$ diagonal channel matrix from the $l^{\text {th }}$ user in the $j^{\text {th }}$ cell to the $c^{\text {th }}$ antenna of the $i^{\text {th }} \mathrm{BS}$.

Assuming each user transmits $d$ data streams, each user selects the same $r \times d$ beamforming matrix $\mathbf{V}$ so that $\hat{\mathbf{x}}_{j l}=$ $\mathbf{V s}_{j l}$ where $\mathbf{s}_{j l}$ is the $d \times 1$ symbol vector to be transmitted. We let $|\mathbf{V}|$ denote the number of columns of $\mathbf{V}$. Note that since each user can access only $r$ dimensions of the total $r N$ dimensions available at any $\mathrm{BS}$, interference alignment is not possible using any set of $N$ users. In order to align interference, we first define a $N|\mathbf{V}|$ dimensional interference space at each of the BSs and then try to align interference from all the out-of-cell users within this $N|\mathbf{V}|$ dimensional space. We set this $N|\mathbf{V}|$ dimensional space to be the space spanned by the columns of the $r N \times N|\mathbf{V}|$ matrix

$$
\mathbf{Q}=\left[\begin{array}{cccc}
\mathbf{V} & \mathbf{0} & \cdots & \mathbf{0} \\
\mathbf{0} & \mathbf{V} & \cdots & \mathbf{0} \\
\vdots & \vdots & \cdots & \mathbf{0} \\
\mathbf{0} & \mathbf{0} & \cdots & \mathbf{V}
\end{array}\right]
$$

In other words, the received signal vector at the $i^{\text {th }}$ BS from every interfering user $(j, l)$ must satisfy

$$
\begin{aligned}
& \operatorname{span}\left(\hat{\mathbf{H}}_{(j l, i)} \mathbf{V}\right) \subseteq \operatorname{span}(\mathbf{Q}) \forall j \neq i \\
& \Rightarrow \operatorname{span}\left(\left[\begin{array}{c}
\hat{\mathbf{H}}_{(j l, i 1)} \mathbf{V} \\
\hat{\mathbf{H}}_{(j l, i 2)} \mathbf{V} \\
\vdots \\
\hat{\mathbf{H}}_{(j l, i N)} \mathbf{V}
\end{array}\right]\right) \subseteq \operatorname{span}(\mathbf{Q}) \forall j \neq i
\end{aligned}
$$

Equivalently, we require

$$
\begin{gathered}
\operatorname{span}\left(\hat{\mathbf{H}}_{(j l, i c)} \mathbf{V}\right) \subseteq \operatorname{span}(\mathbf{V}) \forall i, j \in\{1: G\}, i \neq j, \\
l \in\{1: K\}, c \in\{1: N\} .
\end{gathered}
$$

Noting that all matrices $\hat{\mathbf{H}}_{(j l, i c)}$ are diagonal and hence commute, we adopt the same iterative procedure as outlined in [5] to design the beamforming matrix $\mathbf{V}$. We first set 


$$
\mathbf{V}_{s+1}=\left\{\left(\prod_{\substack{i, j \in\{1: G\}, i \neq j \\ l=1: K, c=1: N}}\left(\hat{\mathbf{H}}_{(j l, i c)}\right)^{\beta_{(j l, i c)}}\right) \mathbf{s} \quad \sum_{\substack{i, j \in\{1: G\}, i \neq j \\ l=1: K, c=1: N}} \beta_{(j l, i c)} \leq s+1, \beta_{(j l, i c)} \in \mathbb{Z}, \beta_{(j l, i c)} \geq 0\right\}
$$

$\mathbf{V}=\mathbf{1}_{r \times 1}$. Then, in each subsequent iteration, we update $\mathbf{V}$ to be the set of interfering vectors received at all the BSs, i.e., if after $s$ iterations, $\mathbf{V}=\mathbf{V}_{s}$, then $\mathbf{V}_{s+1}$ is given by

$$
\begin{aligned}
\mathbf{V}_{s+1}=\left\{\hat{\mathbf{H}}_{j l, i c} \mathbf{V}_{s} \mid\right. & i, j \in\{1: G\}, i \neq j, \\
l & \in\{1: K\}, c \in\{1: N\}\} .
\end{aligned}
$$

A more useful, non-recursive representation of $\mathbf{V}_{s+1}$ is given in (17). Note that in all there are $L=(G-1) G N$ constraints of the form shown in (15). Since the matrices $\hat{\mathbf{H}}_{j l, i c}$ commute, the number of columns in $\mathbf{V}$ after $s$ iterations is given by $\left(\begin{array}{c}s+L \\ s\end{array}\right)$. Thus we see that as the number of iterations $s \rightarrow \infty$,

$$
\frac{\left|\mathbf{V}_{s+1}\right|}{\left|\mathbf{V}_{s}\right|}=\frac{s+L+1}{s+1} \rightarrow 1 \text {. }
$$

This suggests that asymptotically, the column space of the matrix $\mathbf{V}_{s}$ is invariant to linear transformations by the set of matrices $\hat{\mathbf{H}}_{(j l, i c)}$, and hence satisfies the span constraints in (15). Through this procedure we ensure that at each BS, interference from all the out-of-cell users is contained within a sufficiently small number of dimensions $\left(\approx N\left|\mathbf{V}_{s}\right|\right.$ for large $s)$.

We now need to show that (a) the columns of $\mathbf{V}_{s}$ are linearly independent and (b) the received signal from the $K$ users in a cell is separable from the interfering signals at the corresponding BS. We first set the number of channel extensions to be such that $r=\left\lceil\frac{N\left|\mathbf{V}_{s}\right|+K\left|\mathbf{V}_{s}\right|}{N}\right\rceil$ for some large $s$. The columns of $\mathbf{V}_{s}$ can be shown to be linearly independent using Lemma 1 and 2 in [4]. The set of received signals at the $i^{t h}$ BS can also be shown to be linearly independent by considering the received signal matrix

$$
\left[\hat{\mathbf{H}}_{(i 1, i)} \mathbf{V} \hat{\mathbf{H}}_{(i 2, i)} \mathbf{V} \cdots \hat{\mathbf{H}}_{(i K, i)} \mathbf{V}\right]_{r N \times K\left|V_{s}\right|}
$$

and again invoking Lemma 1 and 2 in [4]. We now need to show that the received signal $\hat{\mathbf{H}}_{(i j, i)} \mathbf{V}$ is linearly independent of the columns in $\mathbf{Q}$. We prove this using a contradiction. Suppose this is not true, then there exists a vector $\mathbf{w}$ such that $\left[\left(\hat{\mathbf{H}}_{(i j, i)} \mathbf{V}\right) \mathbf{Q}\right] \mathbf{w}=0 \Rightarrow\left[\left(\hat{\mathbf{H}}_{(i j, i 1)} \mathbf{V}\right) \mathbf{V}\right] \mathbf{w}_{1: r}=0$. Now, since direct channels play no role in the design of $\mathbf{V}$ and because all channels are assumed to be generic, $\left(\hat{\mathbf{H}}_{(i j, i 1)} \mathbf{V}\right)$ is a generic linear transformation of the subspace spanned by $\mathbf{V}$. Hence, the probability that these two subspaces have an overlap in one or more dimensions is almost surely zero, which contradicts our initial assumption. Thus, at all the BSs the received signal from the $K$ users is separable from interference. This shows that $\frac{\left|\mathbf{V}_{s}\right|}{r}=\frac{\left|\mathbf{V}_{s}\right|}{\left\lceil\left(N\left|\mathbf{V}_{s}\right|+K\left|\mathbf{V}_{s}\right|\right) / N\right\rceil} \approx \frac{N}{N+K}$ DoF/user are achievable as $s \rightarrow \infty$.

\section{CONCLUSION}

This paper studies the DoF of MIMO cellular networks with generic, time varying channels. We investigate two-sided and one-sided decomposition of MIMO cellular networks and establish an inner bound on the optimal DoF of such networks. Through a new set of outer bounds, we establish the conditions under which decomposition achieves the optimal DoF of MIMO cellular networks.

\section{REFERENCES}

[1] V. R. Cadambe and S. A. Jafar, "Interference alignment and degrees of freedom of the K-user interference channel," IEEE Trans. Inf. Theory, vol. 54, no. 8, pp. $3425-3441$, Aug. 2008.

[2] C. Suh, M. Ho, and D. N. C. Tse, "Downlink interference alignment," IEEE Trans. Commun., vol. 59, no. 9, pp. 2616-2626, Sep. 2011.

[3] C. Suh and D. N. C. Tse, "Interference alignment for cellular networks," in Proc. Annual Allerton Conf. Commun., Control, and Computing, Sep. 2008, pp. 1037-1044.

[4] V. R. Cadambe and S. A. Jafar, "Interference alignment and the degrees of freedom of wireless X networks," IEEE Trans. Inf. Theory, vol. 55, no. 9 , pp. 3893-3908, 2009.

[5] H. Sun, C. Geng, T. Gou, and S. A. Jafar, "Degrees of freedom of MIMO X networks: Spatial scale invariance, one-sided decomposability and linear feasibility," in Proc. IEEE Int. Symp. Inf. Theory, Jul. 2012, pp. 2082-2086.

[6] A. Ghasemi, A. S. Motahari, and A. K. Khandani, "Interference alignment for the K user MIMO interference channel," in Proc. IEEE Int. Symp. Inf. Theory, Jun. 2010, pp. 360-364.

[7] T. Gou and S. A. Jafar, "Degrees of freedom of the $\mathrm{K}$ user $\mathrm{M} \times \mathrm{N}$ MIMO interference channel," IEEE Trans. Inf. Theory, vol. 56, no. 12, pp. 6040-6057, Dec. 2010.

[8] C. Wang, T. Gou, and S. A. Jafar, "Subspace alignment chains and the degrees of freedom of the three-user MIMO interference channel," IEEE Trans. Inf. Theory, submitted for publication. [Online]. Available: http://arxiv.org/abs/1109.4350

[9] A. S. Motahari, S. O. Gharan, and A. K. Khandani, "Real interference alignment with real numbers." [Online]. Available: http://arxiv.org/abs/0908.1208

[10] T. Kim, D. J. Love, and B. Clerckx, "On the spatial degrees of freedom of multicell and multiuser MIMO channels," IEEE Trans. Inf. Theory, submitted for publication. [Online]. Available: http://arxiv.org/abs/1111.3160

[11] Y. Ma, J. Li, R. Chen, and Q. Liu, "On feasibility of interference alignment for L-cell constant cellular interfering networks," IEEE Commun. Lett., vol. 16, no. 5, pp. $714-716$, May 2012.

[12] S. Ayoughi, M. Nasiri-Kenari, and B. Hossein Khalaj, "On degrees of freedom of the cognitive MIMO two interfering multiple access channels," IEEE Trans. Veh. Technol., vol. PP, no. 99, 2013.

[13] W. Shin, N. Lee, J.-B. Lim, C. Shin, and K. Jang, "On the design of interference alignment scheme for two-cell MIMO interfering broadcast channels," IEEE Trans. Wireless Commun., vol. 10, no. 2, pp. 437 -442, Feb. 2011.

[14] T. Liu and C. Yang, "Interference alignment transceiver design for MIMO interference broadcast channels," in Proc. IEEE Wireless Commun. and Netw. Conf., Apr. 2012.

[15] K. Lee, "Uplink interference alignment for two-cell MIMO interference channels," IEEE Trans. Veh. Technol., vol. PP, no. 99, 2012.

[16] B. Zhuang, R. A. Berry, and M. L. Honig, "Interference alignment in MIMO cellular networks," in Proc. IEEE Int. Conf. Acoustics, Speech and Signal Processing, May 2011.

[17] S.-H. Park and I. Lee, "Degrees of freedom for mutually interfering broadcast channels," IEEE Trans. Inf. Theory, vol. 58, no. 1, pp. 393402, Jan. 2012.

[18] C. Wang, H. Sun, and S. A. Jafar, "Genie chains and the degrees of freedom of the K-user MIMO interference channel," in Proc. IEEE Int. Symp. Inf. Theory, Jul. 2012, pp. 2476-2480. 